TITLE:

\title{
A generalization of Kohnen's estimates for Fourier coefficients of Siegel cusp forms
}

$\operatorname{AUTHOR}(\mathrm{S})$ :

Horie, Taro

\section{CITATION:}

Horie, Taro. A generalization of Kohnen's estimates for Fourier coefficients of Siegel cusp forms. 数理解析研究所講究録 1996, 965: 145-152

ISSUE DATE:

1996-08

URL:

http://hdl.handle.net/2433/60589

RIGHT: 


\title{
A generalization of Kohnen's estimates for Fourier coefficients of Siegel cusp forms
}

\author{
Taro Horie (堀江 太郎) \\ Graduate school of Polymathematics, Nagoya University \\ Chikusa-ku, Nagoya 464-01, Japan \\ E-mail:t-horie@math.nagoya-u.ac.jp
}

The purpose of this article is to show that the main result of $[\mathrm{K}]$ is valid for any level.

Theorem. Let $F$ be a cusp form of integral or half integral weight $k(>2)$ with respect to the subgroup $\Gamma_{2}(N)$ of $\mathrm{Sp}_{2}(\mathbf{Z})$, where

$$
\Gamma_{2}(N):=\left\{\left(\begin{array}{cc}
A & B \\
C & D
\end{array}\right) \in \Gamma_{2} \mid C \equiv 0(\bmod N)\right\}
$$

And let its Fourier expansion be given by

$$
F(Z)=\sum_{T} a(T) \exp (2 \pi i \operatorname{tr} T\langle Z\rangle)
$$

where $T$ runs over positive definite symmetric half-integral $2 \times 2$-matrices. Then we have

$$
a(T) \ll_{\varepsilon, F}(\min T)^{5 / 18+\varepsilon}(\operatorname{det} T)^{(k-1) / 2+\varepsilon} \quad(\forall \varepsilon>0),
$$

where $\min T$ is the smallest positive integer represented by $T$.

The idea to prove Theorem is the same as in [K], that is a combination of appropriate estimates for both Fourier coefficients of Jacobi Poincaré series and Petterson norms of Fourier-Jacobi coefficients of Siegel modular forms.

$\mathcal{H}_{i}$ denotes the Siegel upper half space of degree $i$ consisting of complex $i \times i$-matrices with positive definite imaginary part. We often write

$$
Z=X+i Y=\left(\begin{array}{cc}
\tau & z \\
z & \tau^{\prime}
\end{array}\right)==\left(\begin{array}{cc}
u+i v & x+i y \\
x+i y & u^{\prime}+i v^{\prime}
\end{array}\right) \in \mathcal{H}_{2} .
$$

For simplicity, we condider only the integral weight case.

Proposition 1. We let $\Gamma_{1}^{J}(N)$ be the Jacobi group which is the semi direct product of $\Gamma_{1}(N)$ and $\mathbf{Z}^{2}$, and let $J_{k, m}^{\text {cusp }}(N)$ be the space of holomorphic Jacobi cusp forms on $\mathcal{H}_{1} \times \mathbf{C}$ of weight $k$ and index $m$ with respect to $\Gamma_{1}^{J}(N)$ (cf. e.g. [E-Z]). 
For $\phi$ in $J_{k, m}^{\text {cusp }}(N)$, let $c(n, r)$ be the $(n, r)$-th Fourier coefficient of $\phi\left(n, r \in \mathbf{Z}, r^{2}<\right.$ $4 m n)$. Put $D=r^{2}-4 m n$. Then we have

$$
c(n, r) \ll_{\varepsilon, k}\left(m+|D|^{1 / 2+\varepsilon} ; \frac{|D|^{k / 2-3 / 4}}{m^{(k-1) / 2}}\|\phi\| \quad(\forall \varepsilon>0)\right.
$$

where the constant implied in $\ll$ depends only on $\varepsilon$ and $k$ (not on $m$ ).

Proof. Let $P_{n, r}=P_{k, m, n, r}$ be the $(n, r)$-th Jacobi Poincaré series in $J_{k, m}^{\text {cusp }}(N)$ characterized by

$$
\left\langle\psi, P_{n, r}\right\rangle=\lambda_{k, m, D} b_{n, r}(\psi) \quad\left(\forall \psi \in J_{k, m}^{\text {cusp }}(N)\right)
$$

where $b_{n, r}(\psi)$ denotes the $(n, r)$-th Fourier coefficients of $\psi$ and

$$
\lambda_{k, m, D}:=\frac{1}{2} \Gamma\left(k-\frac{3}{2}\right) \pi^{-k+3 / 2} m^{k-2}|D|^{-k+3 / 2} .
$$

Then the Cauchy-Schwarz inequality gives

$$
|c(n, r)|^{2} \leq \lambda_{k, m, D}^{-2}\|\phi\|^{2}\left\langle P_{n, r}, P_{n, r}\right\rangle=\lambda_{k, m, D}^{-1} b_{n, r}\left(P_{n, r}\right)\|\phi\|^{2} .
$$

We can show that the Fourier coefficient of $P_{n, r}$ as follows (cf. [G-K-Z], p.519);

$$
\begin{gathered}
b_{n, r}\left(P_{n, r}\right)=1+(-1)^{k} \delta_{m}(r)+\frac{i^{k} \tau \sqrt{2}}{\sqrt{m}} \sum_{N \mid c \geq 1} c^{-3 / 2}\left(\exp \left(r^{2} / 2 m c\right) H_{m, c}^{+}(n, r)\right. \\
\left.+(-1)^{k} \exp \left(-r^{2} / 2 m c\right) H_{m, c}^{-}(n, r)\right) J_{k-3 / 2}\left(\frac{\pi|D|}{m c}\right)
\end{gathered}
$$

where

$$
\delta_{m}(r)=\left\{\begin{array}{ll}
1 & \text { if } r \equiv 0(\bmod m) \\
0 & \text { otherwise }
\end{array},\right.
$$

$J_{k-3 / 2}$ is the modified Bessel function of order $k-3 / 2$, and

$$
H_{m, c}^{ \pm}(n, r):=\sum_{x(c), y(c)^{*}} \mathbf{e}_{c}\left(\left(m x^{2}+r x+n\right) \bar{y}+n y \pm r x\right)
$$

where $x$ resp. $y$ run through $\mathbf{Z} / c \mathbf{Z}$ resp. $(\mathbf{Z} / c \mathbf{Z})^{*}, \bar{y}$ denotes an inverse of $y(\bmod c)$, $\mathbf{e}_{c}(b):=\exp (2 \pi i b / c)$ for $c \in \mathbf{N}, b \in \mathbf{Z} / c \mathbf{Z}, \varepsilon(y)=1$ or $i$ according as $y \equiv 1 \quad(\bmod 4)$ or $\equiv 3 \quad(\bmod 4)$, and $\left(\frac{*}{*}\right)$ means the Kronecker symbol. $H_{m, c}^{ \pm}(n, r)$ is a certain character sum, which is Gauss sum for $x$ and Kloosterman sum for $y$, and by factorizing $c$ to prime powers, for $D:=r^{2}-4 m n$ we can prove an estimate

$$
H_{m, c}^{ \pm}(n, r) \ll_{\varepsilon} c^{1+\varepsilon}(D, c) \quad(\forall \varepsilon>0) .
$$

From this and the estimate

$$
J_{k-3 / 2}(x) \ll_{k} \min \left\{x^{-1 / 2}, x^{k-3 / 2}\right\} \quad(x>0)
$$

(cf. e.g. [B], p.4 and p.74), we easily find

$$
b_{n, r}\left(P_{n, r}\right) \ll_{\varepsilon, k} 1+\frac{|D|^{1 / 2+2 \varepsilon}}{m}
$$

for any $\varepsilon>0$ and complete the proof. 
To estimate Petterson norm $\|\phi\|$, for an analogue of the Rankin convolution series

$$
D_{F, F}(s):=\zeta(2 s-2 k+4) \sum_{n \geq 1}\left\langle\phi_{n}, \phi_{n}\right\rangle n^{-s}
$$

where

$$
F(Z)=\sum_{n \geq 1} \phi_{n}(\tau, z) \exp \left(2 \pi i n \tau^{\prime}\right)
$$

we want to use the following Landau's Theorem;

Theorem (Landau-Shintani). Suppose that

$$
\xi(s)=\sum_{n \geq 1} c(n) n^{-s}, \xi_{i}(s)=\sum_{n \geq 1} c_{i}(n) n^{-s}(1 \leq i \leq I)
$$

are Dirichlet serieses with non-negative cefficients which converge for $\operatorname{Re}(s)>\sigma_{0}$, have meromorphic continuation to $\mathbf{C}$ with finitely many poles and satisfy a functional equation

$$
\xi^{*}(\delta-s)=\sum_{i=1}^{I} \xi_{i}^{*}(s)
$$

where

$$
\begin{gathered}
\xi_{i}^{*}(s)=B A^{s} \prod_{j=1}^{J} \Gamma\left(a_{j} s+b_{j}\right) \xi(s) \quad\left(A \in \mathbf{C}, B \in \mathbf{C}, a_{j}>0, b_{j} \in \mathbf{R}\right) \\
\xi_{i}^{*}(s)=B_{i} A_{i}^{s} \prod_{j=1}^{J} \Gamma\left(a_{j} s+b_{j}\right) \xi(s) \quad\left(A_{i} \in \mathbf{C}, B_{i} \in \mathbf{C}, a_{j} \text { and } b_{j} \text { are same as above }\right) .
\end{gathered}
$$

Suppose

$$
\kappa:=\left(2 \sigma_{0}-\delta\right) \sum_{j=1}^{J} a_{j}-\frac{1}{2}>0
$$

Then we have

$$
\sum_{n \leq x} c(n)=\sum_{s: \text { all poles }} \operatorname{Res}\left(\frac{\xi(s)}{s} x^{s}\right)+O_{\eta}\left(x^{\eta}\right)
$$

for any $\eta>\eta_{0}:=\left\{\delta+\sigma_{0}(\kappa-1)\right\} /(\kappa+1)$.

For the proof, see Theorem 3 and its procf in [S-S].

The central extension of $\Gamma_{1}^{J}(N)$ by $\mathbf{Z}$ is ernbedded into $\Gamma_{2}(N)$ via

$$
\left(\left(\begin{array}{ll}
a & b \\
c & d
\end{array}\right), \lambda, \mu, \kappa\right) \mapsto\left(\begin{array}{cccc}
a & 0 & b & \mu^{\prime} \\
\lambda & 1 & \mu & \kappa \\
c & 0 & d & -\lambda^{\prime} \\
0 & 0 & 0 & 1
\end{array}\right), \quad(\lambda, \mu)=\left(\lambda^{\prime}, \mu^{\prime}\right)\left(\begin{array}{cc}
a & b \\
c & d
\end{array}\right)
$$


and we denote by $C_{N}$ the image in $\Gamma_{2}(N)$. Denote the left upper entry of $Z \in \mathcal{H}_{2}$ by $Z_{1}$. For a natural number $N, Z \in \mathcal{H}_{2}$ and $s \in \mathbf{C}$ with $\operatorname{Re}(s) \gg 2$ we define a Klingen-Siegel type Eisenstein series

$$
E_{s, N}(Z):=\sum_{M \in C_{N} \backslash \Gamma_{2}(N)}\left(\frac{\operatorname{det} \operatorname{Im} M\langle Z\rangle}{\operatorname{Im} M\langle Z\rangle_{1}}\right)^{s} .
$$

It is easily seen that this series is well defined, absolutery convergent, and invariant under the action of $\Gamma_{2}(N)$. We put

$$
E_{s, N}^{*}(Z):=\pi^{-s} \Gamma(s) \zeta(2 s) E_{s, N}(Z) .
$$

By Main Lemma on p.545 in [K-S], we know $E_{s, 1}(Z)$ has a meromorphic continuation to $\mathrm{C}$, has only two poles at $s=0,2$ which are simple, and satisfies a functional equation

$$
E_{2-s, 1}^{*}(Z)=E_{s, 1}^{*}(Z) .
$$

By the method of Rankin-Selberg convolution

$$
\pi^{-k+2}\left\langle F E_{s-k+2, N}^{*}, F\right\rangle=D_{F, F}^{*}(s)
$$

can be proved, and analytic properties of $L_{F, F}^{*}(s)$ follow from those of $E_{s, N}^{*}(s)$. But the functional equations are complicated.

The idea to prove Theorem for any level $I N$ is to write the functional equations satisfied by Eisenstein series as a form

$$
E_{2-s, N}^{*}(Z)=\text { a linear combination of } E_{s, m}^{*}(Z)
$$

where $m$ is a natural number with $m \mid N$. This is necessary to apply Rankin's method.

Lemma 1. $E_{s, N}(Z)$ has a meromorphic continuation to $\mathbf{C}$. Its poles are $s=0$ and 2, which are simple. And it satisfies a functior.al equation

$$
E_{2-s, N}^{*}(Z)=\text { a finite sum of } \frac{ \pm n^{s}}{P(s)} E_{s, m}^{*}(Z),
$$

where $m, n$ are natural numbers with $m \mid N$ and $P(s)$ is a finite product of $1-\tilde{m}^{2(2-s)}$ with $\tilde{m} \mid m$.

Proof. For $M=\left(\begin{array}{cc}A & B \\ C & D\end{array}\right)=\left(\begin{array}{cccc}* & * & * & * \\ c_{3} & c_{4} & d_{3} & d_{4}\end{array}\right) \in \Gamma_{2}(N)$, we notice that

$$
\frac{\operatorname{det} \operatorname{Im} M\langle Z\rangle}{\operatorname{Im} M\langle Z\rangle_{1}}=\frac{|Y|}{Y\left[Z^{*}\left(\begin{array}{c}
c_{4} \\
-c_{3}
\end{array}\right)+\left(\begin{array}{c}
d_{4} \\
-d_{3}
\end{array}\right)\right]}
$$

$\left(Y\left[\begin{array}{l}a \\ b\end{array}\right]:=(\bar{a}, \bar{b}) Y\left(\begin{array}{l}a \\ b\end{array}\right), Z^{*}\right.$ means the adjoint matrix of $\left.Z\right)$ and the mapping

$$
\left(\begin{array}{cccc}
* & * & * & * \\
c_{3} & c_{4} & d_{3} & d_{4}
\end{array}\right) \mapsto\left(c_{3}, c_{4}, d_{3}, d_{4}\right)
$$


induces a bijection between

$$
C_{N} \backslash \Gamma_{2}(N) \text { and }\left\{\left(c_{3}, c_{4}, d_{3}, d_{4}\right) \in \mathbf{Z}^{4} \mid \text { primitive and } c_{3} \equiv c_{4} \equiv 0(\bmod N)\right\} \text {. }
$$

In the following sums, $c=\left(\begin{array}{c}c_{3} \\ c_{4}\end{array}\right), d=\left(\begin{array}{c}d_{3} \\ d_{4}\end{array}\right)$ run over $\mathbf{Z}^{2}$. under the condition that $c_{3}, c_{4}, d_{3}, d_{4}$ are relatively prime. In general, for a square free integer $m$ and a natural number $l=p_{1}^{e_{1}} p_{2}^{e_{2}} \ldots p_{r}^{e_{r}} \in \mathbf{N}$ (where $p_{1}, p_{2}, \ldots, p_{r}$ are different prime numbers and $e_{i}>0$ ) it holds

$$
\begin{aligned}
& \frac{1}{l^{s}} E_{s, m}(l Z) \\
& =\sum_{\substack{\left(t, t_{d}\right)=1 \\
c \equiv 0(\bmod m)}} \frac{|Y|^{s}}{\left(Y\left[Z^{*} l c+d\right]\right)^{s}} \\
& =\left(\sum_{\substack{\left.t_{c}, t_{d}\right)=1 \\
\text { al, } t_{d) \neq 1} \\
c=0(\bmod m)}}+\sum_{\substack{\left(t_{c}, t_{d)=1} \\
\left(l, t_{d)=1} \\
c \equiv 0(\bmod m)\right.\right.}}\right) \frac{|Y|^{s}}{\left(Y\left[Z^{*} l c+d\right]\right)^{s}} \\
& =\sum_{\substack{\left.\left(t_{c}, t_{d}\right)=1 \\
d \equiv 0 \bmod \exists p_{i}\right) \\
c \equiv 0(\bmod m)}} \frac{|Y|^{s}}{\left(Y\left[Z^{*} l c+d\right]\right)^{s}}+\sum_{\substack{\left(t_{c}, t_{d}\right)=1 \\
c \equiv 0(\bmod l m)}} \frac{|Y|^{s}}{\left(Y\left[Z^{*} c+d\right]\right)^{s}} \\
& =\sum_{i} \frac{1}{p_{i}^{2 s}} \sum_{\substack{\left.t_{c}, p_{i}^{t} d\right)=1 \\
c \equiv 0(\bmod m)}} \frac{|Y|^{s}}{\left(Y\left[Z^{*}\left(l / p_{i}\right) c+d\right]\right)^{s}} \\
& -\sum_{i \neq j} \frac{1}{\left(p_{i} p_{j}\right)^{2 s}} \sum_{\substack{\left(t_{c}, p_{i} p_{j}^{t} d\right)=1 \\
c \equiv 0(\bmod m)}} \frac{|Y|^{s}}{\left(Y\left[Z^{*}\left(l / p_{i} p_{j}\right) c+d\right]\right)^{s}} \\
& +\ldots \\
& +\sum_{\substack{\left(t_{c}, t_{d, j}=1 \\
c \equiv 0(\bmod l m)\right.}} \frac{|Y|^{s}}{\left(Y\left[Z^{*} c+d\right]\right)^{s}} \\
& =\sum_{i} \frac{1}{p_{i}^{2 s}}\left(\sum_{\substack{\left(t_{c}, t_{d}\right)=1 \\
c \equiv 0(\bmod 0)}}-\sum_{\substack{\left(t_{c}, t_{d}\right)=1 \\
c \equiv 0(\bmod m) \\
c \equiv 0\left(\bmod p_{i}\right)}}\right) \frac{|Y|^{s}}{\left(Y\left[Z^{*}\left(l / p_{i}\right) c+d\right]\right)^{s}} \\
& =\sum_{i} \frac{1}{\left(p_{i}\right)^{2 s}}\left\{E_{s, m}\left(\left(l / p_{i}\right) Z\right)-E_{s, \text { l.c.m. }\left(m, p_{i} ;\right.}\left(\left(l / p_{i}\right) Z\right)\right\} \\
& -\sum_{i \neq j} \frac{1}{\left(l p_{i} p_{j}\right)^{s}}\left\{E_{s, m}\left(\left(l / p_{i} p_{j}\right) Z\right)-E_{s, \text { l.c.m. }\left(m, p_{i}\right)}\left(\left(l / p_{i} p_{j}\right) Z\right)-E_{s, \text { l.c.m. }\left(m, p_{j}\right)}\left(\left(l / p_{i} p_{j}\right) Z\right)\right. \\
& \left.+E_{s, \text { l.c.m. }\left(m, p_{i} p_{j}\right)}\left(\left(l / p_{i} p_{j}\right) Z\right)\right\} \\
& +\ldots
\end{aligned}
$$




$$
\begin{aligned}
& +(-1)^{r-1} \frac{1}{\left(l p_{1} p_{2} \ldots p_{r}\right)^{s}}\left\{E_{s, m}\left(\left(l / p_{1} \ldots p_{r}\right) Z\right)-\sum_{i} E_{s, \text { l.c.m. }\left(m, p_{i}\right)}\left(\left(l / p_{1} \ldots p_{r}\right) Z\right)\right. \\
& \left.\quad+\sum_{i \neq j} E_{s, \text { l.c.m. }\left(m, p_{i} p_{j}\right)}\left(\left(l / p_{1} \ldots p_{r}\right) Z\right)-\ldots+(-1)^{r} E_{s, 1 . c . m .\left(m, p_{1} \ldots p_{r}\right)}\left(\left(l / p_{1} \ldots p_{r}\right) Z\right)\right\} \\
& +E_{s, l m}(Z) .
\end{aligned}
$$

We apply (3) for $m=1$ and $l=N$; if $N$ is not square-free the last term is $E_{s, N}(Z)$, otherwise the last two terms are $\left(-N^{-2 s}+1\right) E_{s, N}(Z)$, and in the both cases the rests are $\pm \tilde{n}^{-s} E_{s, \tilde{m}}(\tilde{l} Z)$ where $\tilde{l}, \tilde{m}, \tilde{n}$ are natural numbers with $\tilde{l} \tilde{m} \mid N, \tilde{m}<N$. Hence for a non-square-free number $N$ we have

$$
E_{s, N}^{*}(Z)=\text { a finite sum of } \pm n^{s} E_{s, m}^{*}(l Z)
$$

where $l, m, n$ are natural numbers with $l m \mid N, m<N$, and for a square-free number $N$ we have

$$
\left(1-N^{2 s}\right) E_{s, N}^{*}(Z)=\text { a finite sum of the same type as above. }
$$

So, by induction on $N$ we deduce that $E_{s, N}(Z)$ has a meromorphic continuation to $\mathbf{C}$, has poles only at $s=0,2$ and satisfies a functional equation

$$
E_{2-s, N}^{*}(Z)=\text { a finite sum of } \frac{ \pm n^{s}}{P_{1}(s)} E_{s, m}^{*}(l Z)
$$

where $l, m, n$ are natural numbers with $l m \mid N$ and $P_{1}(s)$ is a finite product of $1-\tilde{m}^{2(2-s)}$ with $\tilde{m} \mid m$. Now we notice that (3) makes $l$ smaller, and apply (3) repeatedly in all terms in this right-hand side until $l$ becames 1 , then finally we get the functional equation in Lemma 1.

Then we can use Rankin's method and deduce

Lemma 2. Let the notations be as above, and take a natural number $m$ with $m \mid N$. For $L \in \Gamma_{2}$, we write the Fourier expantions of $F\left(L^{-1}\langle Z\rangle\right)$ as

$$
F\left(L^{-1}\langle Z\rangle\right)=\sum_{n \geq 1} \phi_{n: L}(\tau, z) \exp \left(\frac{2 \pi i n \tau^{\prime}}{N}\right) .
$$

We define a Dirichlet series $D_{F, F, m}(s)$ as $\zeta(2 s-2 k+4)$ times

$$
\sum_{n \geq 1}\left\{\sum_{L \in \Gamma_{2}(N) \backslash \Gamma_{2}(m)} \int_{\mathcal{F}}\left|\phi_{n, L}(\tau, z)\right|^{2} \exp \left(-\frac{4 \pi n y^{2}}{v N}\right) v^{k-3} d u d v d x d y\right\} n^{-s}
$$

where $\mathcal{F}$ is a fundamental domain $\Gamma_{1}^{J}(m) \backslash \mathcal{H}_{1} \times \mathbf{C}\left(\right.$ so $\left.D_{F, F, N}(s)=D_{F, F}(s)\right)$, and put

$$
D_{F, F, m}^{*}(s):=(2 \pi)^{-2 s} \Gamma(s) \Gamma(s-k+2) D_{F, F, m}(s) .
$$

Then we have

$$
\pi^{-k+2}\left\langle F E_{s-k+2, m}^{*}, F\right\rangle=N^{s} D_{F, F, m}^{*}(s) .
$$


From (2), (4) and Lemma 1 we have proved

Proposition 2. $D_{F, F, m}(s)$ is a Dirichlet series which has a meromorphic continuation to $\mathbf{C}$, possibly has a unique pole at $s=k$, and satisfies a functional equation

$$
D_{F, F}^{*}(2 k-2-s)=D_{F, F, N}^{*}(2 k-2-s)=\text { a finite sum of } \frac{ \pm n^{s}}{P(s)} D_{F, F, m}^{*}(s)
$$

where $m, n$ are natural numbers with $m \mid N$ and $P(s)$ is a finite product of $1-\tilde{m}^{2(k-s)}$ with $\tilde{m} \mid m$.

Now we can use Landau's Theorem for $D_{F, F, m}(s)$ 's, because $D_{F, F, m}(s) /\left(1-p^{2(k-s)}\right)$ has non-negative coefficients and has a unique pole at $s=k$, hence it converge for $s>k$. Therefore we have

$$
\sum_{n \leq x}\left\|\phi_{n}\right\|^{2}=\left(\operatorname{Res}_{s=k} \frac{D_{F, F}(s)}{s}\right) x^{k}+O_{\varepsilon}\left(x^{k-4 / 9+\varepsilon}\right) \quad(\forall \varepsilon>0)
$$

where $\phi_{n}$ is the $\mathrm{n}$-th Fourier-Jacobi coefficient of $F(Z)$. Taking $x=m$ and $x=m-1$ and subtracting, we find

$$
\left\|\phi_{m}\right\|^{2} \ll_{\varepsilon, F} m^{k-4 / 9+\varepsilon}
$$

hence

$$
\left\|\phi_{m}\right\| \ll_{\epsilon, F} m^{k / 2 i-2 / 9+\varepsilon} \quad(\forall \varepsilon>0) .
$$

By Proposition 2 and (5), we obtain

$$
c(n, r) \ll_{\varepsilon, k}\left(m+|D|^{1 / 2+\varepsilon}\right)^{1 / 2}|D| m^{5 / 18+\varepsilon} .
$$

Both sides of (1) are invariant if $T$ is replaced by ${ }^{t} U T U \quad\left(U \in G L_{2}(\mathbf{Z})\right)$. Hence we may assume that

$$
T=\left(\begin{array}{cc}
n & r / 2 \\
r / 2 & m
\end{array}\right), m=\min T,
$$

so that $a(T)=c(n, r)$. By reduction theory we have $m=\min T \leq \frac{2}{\sqrt{3}}|D|^{1 / 2}$ and complete the proof of Theorem.

\section{Remark.}

1. When $N=1$, the Rankin convolution series $D_{F, F}(s)$ is a linear combination of spinor zeta functions of Hecke eigen forms, as shown in [K-S]. In order to deduce estimates for eigenvalues of Hecke operators, we need find a relation between $D_{F, F, m}(s)$ 's and spinor zeta functions. 
2. When we generalize Kohnen's method to higher genus, we should cut $Z$ as follows;

$$
Z=\left(\begin{array}{ccc|c}
* & \ldots & * & * \\
\vdots & \ddots & \vdots & \vdots \\
* & \ldots & * & * \\
\hline * & \ldots & * & \tau^{\prime}
\end{array}\right)
$$

\section{References}

[B] Bateman, H., Higher Transcendental Functions II, New York-Toront-London, McGrawHill, 1953

[E-Z] Eichler, M., Zagier, D., The Theory of Jacobi Forms (Progress in Maths. vol. 55), Birkhäuser, Boston, (1985)

[G-K-Z] Gross, B., Kohnen, W., and Zagier, D., Heegner points and derivatives of $L$ series. II Math. Ann. 278 (1987), 497-562

[K] Kohnen, W., Estimates for Fourier coefficients of Siegel cusp forms of degree two, Compos. Math. 87 (1993) 231-249

[K2] Kohnen, W., Estimates for Fourier coefficients of Siegel cusp forms of degree two, II, Nagoya Math. J. 128 (1992) 171-176

[K-S] Kohnen, W. and Skoruppa, N.-P., A certain Dirichlet series attached to Siegel modular forms of Degree two, Invent. Math. 95 (1989) 541-558

[S-S] Sato, M., Shintani, T., On zeta functions associated with prehomogeneous vector spaces, Ann. of Math. 100 (1974) 131-170

[T] Tanigawa, Y., Modular descend of Siegel modular forms of half ntegral weight and an analogy of the Maass relation, Nagoya Math. J. 102 (1986) 51-77 